\title{
Extracted Cardiac Lead
}

National Cancer Institute

\section{Source}

National Cancer Institute. Extracted Cardiac Lead. NCI Thesaurus. Code C99952.

The cardiac lead has been removed from the body. (ACC) 